\title{
The Periodic Solutions for Planar 2N-Body Problems
}

\author{
Xiaohong $\mathrm{Hu}^{1,2}$ and Changrong $\mathrm{Zhu}^{3}$ \\ ${ }^{1}$ College of Mathematics, Sichuan University, Chengdu 610064, China \\ ${ }^{2}$ College of Mathematics and Physics, Chongqing University of Posts and Telecommunications, \\ Chongqing 400065, China \\ ${ }^{3}$ Department of Mathematics, Chongqing University, Chongqing 400044, China
}

Correspondence should be addressed to Xiaohong Hu; huxh@cqupt.edu.cn

Received 7 February 2013; Accepted 1 April 2013

Academic Editor: Baodong Zheng

Copyright (C) 2013 X. Hu and C. Zhu. This is an open access article distributed under the Creative Commons Attribution License, which permits unrestricted use, distribution, and reproduction in any medium, provided the original work is properly cited.

Based on the works of Perko and Walter, Moeckel and Simo, and Zhang and Zhou, we study the necessary conditions and suffcient conditions for the uniformly rotating planar nested regular polygonal periodic solutions for the $2 \mathrm{~N}$-body problems.

\section{Introduction and Main Results}

Let $m_{j}>0, q_{j} \in \mathbf{R}^{2}$ be the mass and position of the $j$ th body. The Newtonian $N$-body problem concerns the motion of $N$ point particles. The motion is governed by

$$
m_{j} \ddot{q}_{j}=\frac{\partial U}{\partial q_{j}}, \quad j=1,2, \ldots, N,
$$

where $U$ is the Newtonian potential:

$$
U=\sum_{1 \leq j<k \leq N} \frac{m_{j} m_{k}}{\left|q_{j}-q_{k}\right|}
$$

In the famous paper [1], Perko and Walter proved the following result.

Theorem 1 (see [1]). For $N \geq 4$, the $N$ bodies move with uniformly angular velocity and locate on the vertices of regular $N$-gon if and only if $m_{1}=m_{2}=\cdots=m_{N}$.

In 1995, Moeckel and Simó [2] studied planar nested $2 \mathrm{~N}$ body problems; they assume one regular $N$-gon is inscribed on a unit circle, the other on a circle with radius $a, a>0$ and $a \neq 1$; precisely, let $\rho_{j}=e^{(2 \pi i / N) j}$, where $i^{2}=-1$; and the points $m_{j}$ and $\widetilde{m}_{j}$ locate at $q_{j}$ and $\widetilde{q}_{j}$ :

$$
\begin{gathered}
q_{j}=\left(\rho_{j}-z_{0}\right) e^{i \omega t}, \quad \tilde{q}_{j}=\left(a \rho_{j}-z_{0}\right) e^{i \omega t}, \\
j=1,2, \ldots, N,
\end{gathered}
$$

where $z_{0}=\left(1 /\left(M_{1}+M_{2}\right)\right) \sum\left(m_{j} \rho_{j}+\mathrm{a} \widetilde{m}_{j} \rho_{j}\right), M_{1}=\sum m_{j}$, $M_{2}=\sum \widetilde{m}_{j}$, and $M=M_{1}+M_{2}$. For short, throughout this paper, all indices and summations will range from 1 to $N$ unless we give other restrictions.

For the nested $2 \mathrm{~N}$-body problems, Moeckel and Simó proved the following theorem.

Theorem 2 (see [2]). If $m_{1}=\cdots=m_{N}:=m$ and $\widetilde{m}_{1}=\cdots=$ $\widetilde{m}_{N}:=\widetilde{m}$, then for every mass ratio $r=\widetilde{m} / m$, there are exactly two planar central configurations consisting of two nested regular $\mathrm{N}$-gons. For one of them, the ratio of the sizes of the two nested $N$-gons is less than 1 , and for the other it is greater than 1.

In 2003, Zhang and Zhou [3] studied the inverse problem of Moeckel and Simó's theorem [2], and they got the following results. 
Theorem 3 ([3], the case of $\theta=0)$. If (3) is a solution of (1), then $\omega^{2}$ satisfies

$$
\begin{aligned}
& {\left[\frac{1}{4} \sum_{j \neq N} \csc \frac{\pi j}{N}-\frac{N \omega^{2}}{M}\right] \cdot\left[\frac{1}{4 a^{2}} \sum_{j \neq N} \csc \frac{\pi j}{N}-\frac{a N \omega^{2}}{M}\right]} \\
& \quad=\left[\sum \frac{1-a \rho_{j}}{\left|1-a \rho_{j}\right|^{3}}-\frac{N \omega^{2}}{M}\right] \cdot\left[\sum \frac{a-\rho_{j}}{\left|a-\rho_{j}\right|^{3}}-\frac{a N \omega^{2}}{M}\right]
\end{aligned}
$$

or equivalently

$$
\begin{gathered}
\omega^{2} \\
=\left(\frac{1}{16 a^{2}}\left(\sum_{j \neq N} \csc \frac{\pi j}{N}\right)^{2}\right. \\
\left.-\left(\sum \frac{1-a \rho_{j}}{\left|1-a \rho_{j}\right|^{3}}\right)\left(\sum \frac{a-\rho_{j}}{\left|a-\rho_{j}\right|^{3}}\right)\right) \\
\times\left(\frac{1}{4}\left(a+\frac{1}{a^{2}}\right)\left(\sum_{j \neq N} \csc \frac{\pi j}{N}\right)\right. \\
\left.\left.-a \sum \frac{1-a \rho_{j}}{\left|1-a \rho_{j}\right|^{3}}-\sum \frac{a-\rho_{j}}{\left|a-\rho_{j}\right|^{3}}\right]\right)^{-1} .
\end{gathered}
$$

And in [3], they proved the following theorem [3, Theorem 2]:

Theorem 4. If $\omega$ satisfies (4) or (5) and $\left(q_{1}, \ldots, q_{N} ; \tilde{q}_{1}\right.$, $\left.\ldots, \tilde{q}_{N}\right)$ given by (3) is a periodic solution of (1), then $m_{1}=$ $\cdots=m_{N}$ and $\widetilde{m}_{1}=\cdots=\widetilde{m}_{N}$.

In the proof of [3, Theorem 2], the authors claimed that the first eigenvalue $\lambda_{1}(A B-C D)$ of the matrix $A B-C D$ is simple [3, page 2168]; this is not obvious. In fact, it seems very difficult to prove.

Based on all the above works, we try to give strict proofs about the following two theorems. By the work of Moeckel and Simó [2], if we can get a periodic solution of the form given by (5) with $a \in(0,1)$, the other periodic solution with radius $1 / a$ can be obtained by symmetry. Hence, in the following, we only discuss the periodic solution with radius $a \in(0,1)$. The other one with radius $1 / a$ can be obtained by symmetry.

Theorem 5. For $N \geq 3$, if $a \in\left(a^{*}, 1\right)$, where $a^{*} \in(0,1)$, and $\left(q_{1}, \ldots, q_{N}, \widetilde{q}_{1}, \ldots, \widetilde{q}_{N}\right)$ given by $(3)$ is a periodic solution for (1), then $m_{1}=\cdots=m_{N}$ and $\widetilde{m}_{1}=\cdots=\widetilde{m}_{N}$.
Theorem 6. If $m_{1}=\cdots=m_{N}:=m, \widetilde{m}_{1}=\cdots=\widetilde{m}_{N}:=\widetilde{m}$, and the constant $\omega>0$ is given by

$$
\begin{aligned}
\omega^{2}= & \frac{m}{4} \sum_{j \neq N} \csc \frac{\pi j}{N} \\
& +\widetilde{m} \sum \frac{1-a_{0} \cos (2 \pi j / N)}{\left|1-a_{0} \rho_{j}\right|^{3}},
\end{aligned}
$$

where $\mu=\widetilde{m} / m$ and $a_{0}$ is a unique solution of the following equation:

$$
\begin{aligned}
& \frac{a^{3}-\mu}{4 a^{2}} \sum_{j \neq N} \csc \frac{\pi j}{N} \\
& -(1-\mu) \sum \frac{a}{\left|1-a \rho_{j}\right|^{3}} \\
& +\left(1-a^{2} \mu\right) \sum \frac{\cos (2 \pi j / N)}{\left|1-a \rho_{j}\right|^{3}}=0,
\end{aligned}
$$

then $\left(q_{1}, \ldots, q_{N}, \widetilde{q}_{1}, \ldots, \widetilde{q}_{N}\right)$ with $\left(m_{1}, \ldots, m_{N}, \widetilde{m}_{1}, \ldots, \widetilde{m}_{N}\right)$ is a periodic solution of (1) with angular velocity $\omega$.

When $m_{1}=\cdots=m_{N}$ and $\widetilde{m}_{1}=\cdots=\widetilde{m}_{N}$, we have $z_{0}=0$ which implies that the center of the masses locates at the origin. By Theorem 6, we get a periodic solution of (1) which rotates about the origin with radius $a_{0} \in(0,1)$. By symmetry, (1) has another periodic solution which rotates about the origin with radius $1 / a_{0} \in(1, \infty)$.

\section{The Proof of Theorem 5}

Substituting (3) into (1), we have

$$
\begin{aligned}
& -\omega^{2}\left(\rho_{k}-z_{0}\right) e^{i \omega t}=\sum_{j \neq k} \frac{m_{j}\left(q_{j}-q_{k}\right)}{\left|q_{j}-q_{k}\right|^{3}}+\sum \frac{\tilde{m}_{j}\left(\tilde{q}_{j}-q_{k}\right)}{\left|\tilde{q}_{j}-q_{k}\right|^{3}}, \\
& -\omega^{2}\left(a \rho_{k}-z_{0}\right) e^{i \omega t}=\sum \frac{m_{j}\left(q_{j}-\tilde{q}_{k}\right)}{\left|q_{j}-\tilde{q}_{k}\right|^{3}}+\sum_{j \neq k} \frac{\widetilde{m}_{j}\left(\tilde{q}_{j}-\tilde{q}_{k}\right)}{\left|\tilde{q}_{j}-\tilde{q}_{k}\right|^{3}},
\end{aligned}
$$

where $k=1,2, \ldots, N$.

By (3), (8) can be written as

$$
\begin{aligned}
-\omega^{2}\left(\rho_{k}-z_{0}\right) & =\sum_{j \neq k} \frac{m_{j}\left(\rho_{j}-\rho_{k}\right)}{\left|\rho_{j}-\rho_{k}\right|^{3}}+\sum \frac{\widetilde{m}_{j}\left(a \rho_{j}-\rho_{k}\right)}{\left|a \rho_{j}-\rho_{k}\right|^{3}}, \\
-\omega^{2}\left(a \rho_{k}-z_{0}\right) & =\sum \frac{m_{j}\left(\rho_{j}-a \rho_{k}\right)}{\left|\rho_{j}-a \rho_{k}\right|^{3}}+\frac{1}{a^{2}} \sum_{j \neq k} \frac{\widetilde{m}_{j}\left(\rho_{j}-\rho_{k}\right)}{\left|\rho_{j}-\rho_{k}\right|^{3}},
\end{aligned}
$$


where $k=1,2, \ldots, N$. Equation (9) is equivalent to

$$
\begin{aligned}
\omega^{2} \rho_{k}= & \left(\sum_{j \neq k} \frac{\rho_{k}-\rho_{j}}{\left|\rho_{k}-\rho_{j}\right|^{3}} m_{j}+\frac{\omega^{2}}{M} \sum \rho_{j} m_{j}\right) \\
& +\left(\sum \frac{\rho_{k}-a \rho_{j}}{\left|\rho_{k}-a \rho_{j}\right|^{3}} \widetilde{m}_{j}+\frac{a \omega^{2}}{M} \sum \rho_{j} \widetilde{m}_{j}\right), \\
a \omega^{2} \rho_{k}= & \left(\sum \frac{a \rho_{k}-\rho_{j}}{\left|a \rho_{k}-\rho_{j}\right|^{3}} m_{j}+\frac{\omega^{2}}{M} \sum \rho_{j} m_{j}\right) \\
& +\left(\frac{1}{a^{2}} \sum_{j \neq k} \frac{\rho_{k}-\rho_{j}}{\left|\rho_{k}-\rho_{j}\right|^{3}} \widetilde{m}_{j}+\frac{a \omega^{2}}{M} \sum \rho_{j} \widetilde{m}_{j}\right),
\end{aligned}
$$

where $k=1,2, \ldots, N$. Multiplying both sides of (10) by $\rho_{-k}$, we get

$$
\begin{aligned}
\omega^{2}= & \left(\sum_{j \neq k} \frac{1-\rho_{j-k}}{\left|1-\rho_{j-k}\right|^{3}} m_{j}+\frac{\omega^{2}}{M} \sum \rho_{j-k} m_{j}\right) \\
& +\left(\sum \frac{1-a \rho_{j-k}}{\left|1-a \rho_{j-k}\right|^{3}} \widetilde{m}_{j}+\frac{a \omega^{2}}{M} \sum \rho_{j-k} \widetilde{m}_{j}\right), \\
a \omega^{2}= & \left(\sum \frac{a-\rho_{j-k}}{\left|a-\rho_{j-k}\right|^{3}} m_{j}+\frac{\omega^{2}}{M} \sum \rho_{j-k} m_{j}\right) \\
& +\left(\frac{1}{a^{2}} \sum_{j \neq k} \frac{1-\rho_{j-k}}{\left|1-\rho_{j-k}\right|^{3}} \widetilde{m}_{j}+\frac{a \omega^{2}}{M} \sum \rho_{j-k} \widetilde{m}_{j}\right),
\end{aligned}
$$

where $k=1,2, \ldots, N$.

Let $A=\left(a_{k, j}\right)_{N \times N}, B=\left(b_{k, j}\right)_{N \times N}, C=\left(c_{k, j}\right)_{N \times N}$, and $D=$ $\left(d_{k, j}\right)_{N \times N}$, where

$$
\begin{gathered}
a_{k, j}:=\left\{\begin{array}{l}
\frac{1-\rho_{j-k}}{\left|1-\rho_{j-k}\right|^{3}}, \quad \text { if } j \neq k, \\
0, \quad \text { otherwise, }
\end{array}\right. \\
b_{k, j}:=\frac{1-a \rho_{j-k}}{\left|1-a \rho_{j-k}\right|^{3}}, \\
c_{k, j}:=\frac{a-\rho_{j-k}}{\left|a-\rho_{j-k}\right|^{3}}, \\
d_{k, j}:=\rho_{j-k} .
\end{gathered}
$$

Then (11) can be rewritten in the following compact form:

$$
\begin{gathered}
\left(A+\frac{\omega^{2}}{M} D\right) \vec{m}+\left(B+\frac{a \omega^{2}}{M} D\right) \overrightarrow{\vec{m}}=\omega^{2} \overrightarrow{1}, \\
\left(C+\frac{\omega^{2}}{M} D\right) \vec{m}+\left(\frac{1}{a^{2}} A+\frac{a \omega^{2}}{M} D\right) \overrightarrow{\vec{m}}=a \omega^{2} \overrightarrow{1},
\end{gathered}
$$

where $\vec{m}=\left(m_{1}, \ldots, m_{N}\right), \overrightarrow{\vec{m}}=\left(\widetilde{m}_{1}, \ldots, \widetilde{m}_{N}\right)$, and $\overrightarrow{1}=(1$, $\ldots, 1)$.

An $N \times N$ matrix $\mathscr{R}=\left(r_{j, k}\right)_{N \times N}$ is called circulant (see [4]) if

$$
r_{j, k}=r_{j-1, k-1},
$$

where $r_{0, k}$ and $r_{j, 0}$ are equal to $r_{N, k}$ and $r_{j, N}$, respectively. Let $\rho_{0}=1$. If $\mathscr{R}$ is a circulant matrix, its general formulas for the eigenvalues $\lambda_{k}(\mathscr{R})$ and eigenvectors $v_{k}(\mathscr{R})$ are

$$
\begin{gathered}
\lambda_{k}(\mathscr{R})=\sum_{j} r_{1, j} \rho_{k-1}^{j-1}, \quad k=1,2, \ldots, N, \\
v_{k}(\mathscr{R})=\left(\rho_{k-1}, \rho_{k-1}^{2}, \ldots, \rho_{k-1}^{N}\right), \quad k=1,2, \ldots, N .
\end{gathered}
$$

Remark 7. From the formula (15), we have $\lambda_{1}(\mathscr{R})=\sum_{j=1}^{N} r_{1, j}$. The last equation implies that the eigenvalue $\lambda_{1}(\mathscr{R})$ is equal to the summation of the first row of matrix $\mathscr{R}$; thus, we can get that the summation of any row, and hence, the summation of any column is equal to $\lambda_{1}(\mathscr{R})$.

According to the definition of circulant matrix, it is easy to check that the matrixes $A+\left(\omega^{2} / M\right) D, B+\left(a \omega^{2} / M\right) D, C+$ $\left(\omega^{2} / M\right) D$, and $\left(1 / a^{2}\right) A+\left(a \omega^{2} / M\right) D$ are circulant. For convenience, we introduce some notations. Let $\lambda_{k}^{(1)}, \lambda_{k}^{(2)}, \lambda_{k}^{(3)}$, and $\lambda_{k}^{(4)}$ be the $k$ th eigenvalue of matrixes $A+\left(\omega^{2} / M\right) D, B+$ $\left(a \omega^{2} / M\right) D, C+\left(\omega^{2} / M\right) D$, and $\left(1 / a^{2}\right) A+\left(a \omega^{2} / M\right) D$, respectively. Then we have the following.

Proposition 8. All of the eigenvalues of matrixes $A+\left(\omega^{2} / M\right) D$, $B+\left(a \omega^{2} / M\right) D, C+\left(\omega^{2} / M\right) D$, and $\left(1 / a^{2}\right) A+\left(a \omega^{2} / M\right) D$ are real.

Proof. We only give the proof for the matrix $A+\left(\omega^{2} / M\right) D$. The proofs for the rest are similar. Since $A+\left(\omega^{2} / M\right) D$ is circulant and $\left(\omega^{2} / M\right)$ is real number, we get from (12) that

$$
\begin{aligned}
\bar{a}_{1, j} & +\frac{\omega^{2}}{M} \bar{d}_{1, j} \\
& =\frac{1-\bar{\rho}_{j-1}}{\left|1-\bar{\rho}_{j-1}\right|^{3}}+\frac{\omega^{2}}{M} \bar{\rho}_{j-1} \\
& =a_{1, N-j+2}+\frac{\omega^{2}}{M} d_{1, N-j+2},
\end{aligned}
$$

where $j=1,2, \ldots, N$. Thus, the matrix $A+\left(\omega^{2} / M\right) D$ is Hermitian. We know that all the eigenvalues of $A+\left(\omega^{2} / M\right) D$ are real since the eigenvalues of Hermitian matrix are real. The proof is completed.

From the proof of Proposition 8, we have known that $A+\left(\omega^{2} / M\right) D, B+\left(a \omega^{2} / M\right) D, C+\left(\omega^{2} / M\right) D$, and $\left(1 / a^{2}\right) A+$ $\left(a \omega^{2} / M\right) D$ are Hermitian. Thus, the vectors $\left\{v_{1}, \ldots, v_{N}\right\}$ defined by (16) are basis of $\mathbf{C}^{N}$. It is clear that $\vec{m} \in \mathbf{C}^{N}$ and $\overrightarrow{\vec{m}} \in \mathbf{C}^{N}$. Let

$$
\vec{m}=a_{1} v_{1}+\cdots+a_{N} v_{N}, \quad \overrightarrow{\vec{m}}=b_{1} v_{1}+\cdots+b_{N} v_{N},
$$


where $a_{j}, b_{j} \in$ C. Substituting (18) into (13), we can get

$$
\begin{gathered}
\omega^{2} \overrightarrow{1}=\sum a_{j} \lambda_{j}^{(1)} v_{j}+\sum b_{j} \lambda_{j}^{(2)} v_{j}, \\
a \omega^{2} \overrightarrow{1}=\sum a_{j} \lambda_{j}^{(3)} v_{j}+\sum b_{j} \lambda_{j}^{(4)} v_{j} .
\end{gathered}
$$

Note that $v_{1}=\overrightarrow{1}$. We can get from (19) that

$$
\begin{aligned}
& \left(a_{1} \lambda_{1}^{(1)}+b_{1} \lambda_{1}^{(2)}-\omega^{2}\right) v_{1}+\sum_{j=2}\left(a_{j} \lambda_{j}^{(1)}+b_{j} \lambda_{j}^{(2)}\right) v_{j}=0 \\
& \left(a_{1} \lambda_{1}^{(3)}+b_{1} \lambda_{1}^{(4)}-a \omega^{2}\right) v_{1}+\sum_{j=2}\left(a_{j} \lambda_{j}^{(3)}+b_{j} \lambda_{j}^{(4)}\right) v_{j}=0 .
\end{aligned}
$$

Since $v_{1}, v_{2}, \ldots, v_{N}$ are basis, we can get from (20) that

$$
\begin{gathered}
a_{1} \lambda_{1}^{(1)}+b_{1} \lambda_{1}^{(2)}=\omega^{2}, \\
a_{1} \lambda_{1}^{(3)}+b_{1} \lambda_{1}^{(4)}=a \omega^{2}, \\
a_{j} \lambda_{j}^{(1)}+b_{j} \lambda_{j}^{(2)}=0, \\
a_{j} \lambda_{j}^{(3)}+b_{j} \lambda_{j}^{(4)}=0, \quad j=2,3, \ldots, N .
\end{gathered}
$$

Lemma 9. If $\lambda_{j}^{(1)} \lambda_{j}^{(4)}-\lambda_{j}^{(2)} \lambda_{j}^{(3)} \neq 0, j=1,2, \ldots, N-1$, then $m_{1}=m_{2}=\cdots=m_{N}$ and $\widetilde{m}_{1}=\widetilde{m}_{2}=\cdots=\widetilde{m}_{N}$.

Proof

Case 1 (if $\lambda_{N}^{(1)} \lambda_{N}^{(4)}-\lambda_{N}^{(2)} \lambda_{N}^{(3)} \neq 0$ ). It is clear that

$$
\lambda_{j}^{(1)} \lambda_{j}^{(4)}-\lambda_{j}^{(2)} \lambda_{j}^{(3)}=\left|\begin{array}{ll}
\lambda_{j}^{(1)} & \lambda_{j}^{(2)} \\
\lambda_{j}^{(3)} & \lambda_{j}^{(4)}
\end{array}\right| \neq 0, \quad j=1,2, \ldots, N .
$$

By Gramer's rule, we get from (22) and (23) that

$$
a_{2}=\cdots=a_{N}=b_{2}=\cdots=b_{N}=0 \text {. }
$$

Note that $\omega^{2}$ and $a \omega^{2}$ are real. From Proposition 8 , it is clear that $\lambda_{1}^{(1)}, \lambda_{1}^{(2)}, \lambda_{1}^{(3)}$, and $\lambda_{1}^{(4)}$ are real. Thus, we know from (21) and (23) for $j=1$ that $a_{1}$ and $b_{1}$ are real. Substituting (24) into (18), we get

$$
\vec{m}=a_{1} v_{1}, \quad \overrightarrow{\tilde{m}}=b_{1} v_{1}
$$

Thus, we have

$$
m_{1}=m_{2}=\cdots=m_{N}, \quad \widetilde{m}_{1}=\cdots=\widetilde{m}_{N} .
$$

Case 2. (if $\lambda_{N}^{(1)} \lambda_{N}^{(4)}-\lambda_{N}^{(2)} \lambda_{N}^{(3)}=0$ ). By the similar proof as to (24), we have

$$
a_{2}=\cdots=a_{N-1}=b_{2}=\cdots=b_{N-1}=0 .
$$

We can get from (18) and (27) that

$$
\vec{m}=a_{1} v_{1}+a_{N} v_{N}, \quad \overrightarrow{\vec{m}}=b_{1} v_{1}+b_{N} v_{N}
$$

Since $a_{1}$ and $b_{1}$ are real, it follows from (28) that $\vec{m} \in \mathbf{R}^{N}$ if and only if $a_{N}=0$ or $a_{N} v_{N} \in \mathbf{R}^{N}$. If $a_{N} v_{N} \in \mathbf{R}^{N}$, from the general formulas of eigenvectors defined in (16), we know that

$$
v_{N}=\left(\rho_{N-1}, \rho_{N-1}^{2}, \ldots, \rho_{N-1}^{N-1}, 1\right) .
$$

Since $a_{N} v_{N}=\left(a_{N} \rho_{N-1}, \ldots, a_{N} \rho_{N-1}^{N-1}, a_{N}\right) \in \mathbf{R}^{N}$, so $a_{N} \in \mathbf{R}$. Hence, $\vec{m} \in \mathbf{R}^{N}$ if and only if $a_{N}=0$ or $v_{N} \in \mathbf{R}^{N}$. If $v_{N} \in \mathbf{R}^{N}$, then $\rho_{N-1} \in \mathbf{R}$ from (29), which implies that $\sin (2 \pi(N-$ $1) / N)=-\sin (2 \pi / N)=0$. But it is impossible for $N \geq 3$. Thus, $a_{N}=0$. Similarly, we can get $b_{N}=0$. We get from (28) that

$$
\vec{m}=a_{1} v_{1}, \quad \overrightarrow{\vec{m}}=b_{1} v_{1} \text {. }
$$

Thus, we have

$$
m_{1}=\cdots=m_{N}, \quad \widetilde{m}_{1}=\cdots=\widetilde{m}_{N} .
$$

From Cases 1 and 2, the proof is completed.

The rest of the proof is to verify the assumptions of Lemma 9 by the special structure of our matrixes (12). In order to proceed the proof, we must study the eigenvalues in more details. Since $\rho_{j}$ is the root of unity, it is easy to check that

$$
\sum_{j} \rho_{j}^{k}= \begin{cases}0, & \text { if } k \neq N \\ N, & \text { if } k=N\end{cases}
$$

Then from the general formulas of eigenvalue of circulant matrix, we have

$$
\begin{aligned}
\lambda_{k}^{(1)} & =\sum_{j}\left(a_{1, j}+\frac{\omega^{2}}{M} d_{1, j}\right) \rho_{j-1}^{k-1} \\
& =\sum_{j \neq 1} \frac{\rho_{j-1}^{k-1}-\rho_{j-1}^{k}}{\left|1-\rho_{j-1}\right|^{3}} \\
& =\sum_{j \neq N} \frac{\rho_{j}^{k-1}-\rho_{j}^{k}}{\left|1-\rho_{j}\right|^{3}},
\end{aligned}
$$

where $k=1,2, \ldots, N-1$ and (32) is used. From Proposition 8, we know that the eigenvalue $\lambda_{k}^{(1)}$ is real. Thus, we only take the real part and get that

$$
\lambda_{k}^{(1)}=\sum_{j \neq N} \frac{\cos (2 \pi j / N)(k-1)-\cos (2 \pi j / N) k}{\left|1-\rho_{j}\right|^{3}},
$$


Note that $\left|a-\rho_{j}\right|=\left|1-a \rho_{j}\right|$. Using similar method as proving (34), we have

$$
\begin{aligned}
\lambda_{k}^{(2)} & =\sum\left(b_{1 j}+\frac{a \omega^{2}}{M} d_{1 j}\right) \rho_{j-1}^{k-1} \\
& =\sum \frac{\cos (2 \pi j / N)(k-1)-a \cos (2 \pi j / N) k}{\left|1-a \rho_{j}\right|^{3}}, \\
\lambda_{k}^{(3)} & =\sum\left(c_{1 j}+\frac{\omega^{2}}{M} d_{1 j}\right) \rho_{j-1}^{k-1} \\
& =\sum \frac{\operatorname{acos}(2 \pi j / N)(k-1)-\cos (2 \pi j / N) k}{\left|1-a \rho_{j}\right|^{3}}, \\
\lambda_{k}^{(4)} & =\sum\left(\frac{1}{a^{2}} a_{1 j}+\frac{a \omega^{2}}{M} d_{1 j}\right) \rho_{j-1}^{k-1} \\
& =\frac{1}{a^{2}} \sum_{j \neq N} \frac{\cos (2 \pi j / N)(k-1)-\cos (2 \pi j / N) k}{\left|1-\rho_{j}\right|^{3}}
\end{aligned}
$$

where $k=1,2, \ldots, N-1$.

Lemma 10. $\lambda_{k}^{(1)} \lambda_{k}^{(4)}-\lambda_{k}^{(2)} \lambda_{k}^{(3)}=\lambda_{N-k+1}^{(1)} \lambda_{N-k+1}^{(4)}-\lambda_{N-k+1}^{(2)} \lambda_{N-k+1}^{(3)}$, $k=2, \ldots, N-1$.

Proof. We get from (34) that

$$
\begin{aligned}
& \lambda_{N-k+1}^{(1)} \\
& \quad=\sum_{j \neq N} \frac{\cos (2 \pi j / N) k-\cos (2 \pi j / N)(k-1)}{\left|1-\rho_{j}\right|^{3}} \\
& \quad=-\lambda_{k}^{(1)}, \quad \text { for } k=2,3, \ldots, N-1 .
\end{aligned}
$$

Similarly, we get from (35) that

$$
\begin{aligned}
& \lambda_{N-k+1}^{(2)}=-\lambda_{k}^{(3)}, \\
& \lambda_{N-k+1}^{(3)}=-\lambda_{k}^{(2)}, \\
& \lambda_{N-k+1}^{(4)}=-\lambda_{k}^{(4)},
\end{aligned}
$$

where $k=2,3, \ldots, N-1$. From (36) and (37), the result follows.

Remark 11. Let

$$
\ell= \begin{cases}\frac{N}{2}, & \text { if } N \text { is even, } \\ \frac{(N+1)}{2}, & \text { if } N \text { is odd. }\end{cases}
$$

From Lemma 10, it is clear that to prove all the $N-1$ inequalities of Lemma 9 suffices to prove the $\ell$ inequalities $\lambda_{k}^{(1)} \lambda_{k}^{(4)}-\lambda_{k}^{(2)} \lambda_{k}^{(3)} \neq 0, k=1,2, \ldots, \ell$.

With the similar proof as in [2, Lemma 2], we can get the following proposition.
Proposition 12. Let $\psi_{\beta, k}(a)=\sum_{j}\left(\cos (2 \pi j / N) k /\left|1-a \rho_{j}\right|^{\beta}\right)$. Then for $k=1,2, \ldots, N, \beta>0$ and $a \in(0,1), \psi_{\beta, k}(a)$ and all of its derivatives are positive.

Lemma 13. For $N \geq 3, \lambda_{1}^{(1)} \lambda_{1}^{(4)}-\lambda_{1}^{(2)} \lambda_{1}^{(3)}>0$.

Proof. From (34) and (35), we can get

$$
\begin{aligned}
& \lambda_{1}^{(1)}=\frac{1}{4} \sum_{j \neq N} \csc \frac{\pi j}{N}, \\
& \lambda_{1}^{(2)}=\sum \frac{1-a \cos (2 \pi j / N)}{\left|1-a \rho_{j}\right|^{3}}, \\
& \lambda_{1}^{(3)}=\sum \frac{a-\cos (2 \pi j / N)}{\left|1-a \rho_{j}\right|^{3}}, \\
& \lambda_{1}^{(4)}=\frac{1}{4 a^{2}} \sum_{j \neq N} \csc \frac{\pi j}{N} .
\end{aligned}
$$

Let $A_{1}(a)=\lambda_{1}^{(1)} \lambda_{1}^{(4)}-\lambda_{1}^{(2)} \lambda_{1}^{(3)}$. Then

$$
\begin{aligned}
A_{1}(a)= & \frac{1}{16 a^{2}}\left(\sum_{j \neq N} \csc \frac{\pi j}{N}\right)^{2} \\
& -\left(\sum \frac{1-a \cos (2 \pi j / N)}{\left|1-a \rho_{j}\right|^{3}}\right) \\
& \times\left(\sum \frac{a-\cos (2 \pi j / N)}{\left|1-a \rho_{j}\right|^{3}}\right) \\
= & \frac{1}{16 a^{2}}\left(\sum_{j \neq N} \csc \frac{\pi j}{N}\right)^{2} \\
& -a\left(\sum \frac{1}{\left|1-a \rho_{j}\right|^{3}}\right)^{2}-a\left(\sum \frac{\cos (2 \pi j / N)}{\left(1-a \rho_{j}\right)^{3}}\right)^{2} \\
& +\left(1+a^{2}\right)\left(\sum \frac{\cos (2 \pi j / N)}{\left|1-a \rho_{j}\right|^{3}}\right)
\end{aligned}
$$

Clearly, $\sum\left(1 /\left|1-a \rho_{j}\right|^{3}\right)>0$. By Proposition 12 , we get that $\psi_{3,1}=\sum\left(\cos (2 \pi j / N) /\left|1-a \rho_{j}\right|^{3}\right)>0$ for $a \in(0,1)$. Note that $(1 / 4) \sum_{j \neq N} \csc (\pi j / N)=\sum_{j \neq N}\left((1-\cos (2 \pi j / N)) /\left|1-\rho_{j}\right|^{3}\right)>$ 0 . We have from (40)

$$
\begin{aligned}
A_{1}(a)> & \frac{1}{16 a^{2}}\left(\sum_{j \neq N} \csc \frac{\pi j}{N}\right)^{2} \\
& -a\left(\sum \frac{1}{\left|1-a \rho_{j}\right|^{3}}\right)^{2}-a\left(\sum \frac{\cos (2 \pi j / N)}{\left|1-a \rho_{j}\right|^{3}}\right)^{2}
\end{aligned}
$$




$$
\begin{aligned}
& +2 a\left(\sum \frac{1}{\left|1-a \rho_{j}\right|^{3}}\right)\left(\sum \frac{\cos (2 \pi j / N)}{\left|1-a \rho_{j}\right|^{3}}\right) \\
= & \frac{1}{16 a^{2}}\left(\sum_{j \neq N} \csc \frac{\pi j}{N}\right)^{2} \\
& -a\left(\sum_{\left|1-a \rho_{j}\right|^{3}}-\sum \frac{1}{\left|1-a \rho_{j}\right|^{3}}\right)^{2} \\
= & \frac{1}{a^{2}}\left\{\sum_{j \neq N} \frac{1-\cos (2 \pi j / N)}{\left|1-\rho_{j}\right|^{3}}\right. \\
& \left.-\sum_{j \neq N} \frac{a^{3 / 2}(1-\cos (2 \pi j / N))}{\left|1-a \rho_{j}\right|^{3}}\right\} \cdot g(a) \\
= & \frac{1}{a^{2}}\left\{\sum_{j \neq N}\left(1-\cos \frac{2 \pi j}{N}\right) \cdot h(j, a)\right\} \cdot g(a),
\end{aligned}
$$

where $g(a)=(1 / 4) \sum_{j \neq N} \csc (\pi j / N)+\sum_{j \neq N}\left(a^{3 / 2}(1-\right.$ $\left.\cos (2 \pi j / N)) /\left|1-a \rho_{j}\right|^{3}\right)$ and $h(j, a)=\left(1 /\left|1-\rho_{j}\right|^{3}\right)-\left(a^{3 / 2} / \mid 1-\right.$ $\left.\left.a \rho_{j}\right|^{3}\right)$. Note that $h(j, a)=\left(1 /\left|1-\rho_{j}\right|^{3}\right)-\left(a^{3 / 2} /\left(1+a^{2}-\right.\right.$ $\left.2 a \cos (2 \pi j / N))^{3 / 2}\right)$ and hence

$$
\frac{d h(j, a)}{d a}=\frac{(3 / 2) a^{1 / 2}(a-1)(a+1)}{\left|1-a \rho_{j}\right|^{5}}<0, \quad \text { for } a \in(0,1) .
$$

We know from (42) that $h(j, a)$ is decreasing function in $a$ for $a \in(0,1)$. It is easy to check that $h(j, 1)=0$. Thus

$$
h(j, a)>h(j, 1)=0, \quad \text { for } a \in(0,1), j=1, \ldots, N-1 .
$$

So, $\sum_{j \neq N}(1-\cos (2 \pi j / N)) \cdot h(j, a)>0$ since $h(j, a)>0$ and $1-\cos (2 \pi j / N)>0$. Note that $g(a)>0$ for $a \in(0,1)$. From (41), we can get $A_{1}(a)>0$. The result follows.

For $k=1,2, \ldots, \ell$, let

$$
\alpha_{k}(a)=\sum_{j \neq N} \frac{\cos (2 \pi j(k-1) / N)-\cos (2 \pi j k / N)}{\left|1-a \rho_{j}\right|^{3}} .
$$

Lemma 14. If $N$ is odd, then $\alpha_{(N+1) / 2}(1)=0$; If $N$ is even, then $\alpha_{N / 2}(1)>0$.
Proof. If $N$ is odd, by the definition of $\alpha_{k}$, we have

$$
\begin{aligned}
& \alpha_{(N+1) / 2}(1) \\
& =\sum_{j \neq N}\left(\cos \frac{2 \pi j(((N+1) / 2)-1)}{N}\right. \\
& \left.\quad \quad-\cos \frac{2 \pi j((N+1) / 2)}{N}\right) \\
& \quad \times\left(\left|1-\rho_{j}\right|^{3}\right)^{-1} \\
& =\sum_{j \neq N} \frac{\cos (\pi j-(\pi j / N))-\cos (\pi j+(\pi j / N))}{\left|1-\rho_{j}\right|^{3}}=0 .
\end{aligned}
$$

If $N$ is even, we get that

$$
\begin{aligned}
& \alpha_{N / 2}(1) \\
&= \sum_{j \neq N} \frac{\cos (2 \pi j((N / 2)-1) / N)-\cos (2 \pi j(N / 2) / N)}{\left|1-\rho_{j}\right|^{3}} \\
&= \frac{1}{4} \sum_{j \neq N}(-1)^{j+1} \frac{1}{\sin (\pi j / N)} \\
&=\frac{1}{4}\left[\sum_{j=1}^{N / 2}(-1)^{j+1} \frac{1}{\sin (\pi j / N)}\right. \\
&\left.\quad+\sum_{j=1}^{N / 2-1}(-1)^{j+1} \frac{1}{\sin (\pi j / N)}\right] .
\end{aligned}
$$

Case 1. If $N / 2$ is even, then

$$
\alpha_{N / 2}(1)=\frac{1}{4}\left[2 \sum_{j=1}^{N / 2}(-1)^{j+1} \frac{1}{\sin (\pi j / N)}+1\right] .
$$

Since the signs of the terms in the sum $\sum_{j=1}^{N / 2}(-1)^{j+1}(1 /$ $\sin (\pi j / N))$ alternate and $1 / \sin (\pi j / N)$ is decreasing in $j$ for $1 \leq j \leq N / 2$, we get that $\sum_{j=1}^{N / 2}(-1)^{j+1}(1 / \sin (\pi j / N))>0$ and hence $\alpha_{N / 2}(1)>0$.

Case 2. If $N / 2$ is odd, then

$$
\alpha_{N / 2}(1)=\frac{1}{4}\left[2 \sum_{j=1}^{N / 2-1}(-1)^{j+1} \frac{1}{\sin (\pi j / N)}+1\right] .
$$

Since the signs of the terms in summation $\sum_{j=1}^{N / 2-1}(-1)^{j+1}$ $(1 / \sin (\pi j / N))$ alternate and $1 / \sin (\pi j / N)$ is decreasing in $j$ for $1 \leq j \leq N / 2-1$, we get that $\sum_{j=1}^{(N / 2)-1}(-1)^{j+1}(1 /$ $\sin (\pi j / N))>0$ and hence $\alpha_{N / 2}(1)>0$.

By Cases 1 and 2, we see that $\alpha_{N / 2}(1)>0$ for even $N$. 
Proposition 15. For $k=1,2, \ldots, \ell-1$, then $\alpha_{k}(1)>0$.

Proof. Clearly, $\alpha_{1}(1)>0$. We need to prove $\alpha_{k}(1)>0, k \geq 2$. Note that

$$
\begin{aligned}
& \frac{2}{\sin (\pi j / N)} \sum_{m=0}^{k-1} \cos \frac{2 \pi j}{N} m \\
& =\frac{2 \sin (\pi j k / N) \cos (\pi j(k-1) / N)}{\sin ^{2}(\pi j / N)} \\
& =\frac{\sin (\pi j(2 k-1) / N)}{\sin ^{2}(\pi j / N)}+\frac{1}{\sin (\pi j / N)} .
\end{aligned}
$$

Hence, by the definition of $\alpha_{k}(a)$, we have

$$
\begin{aligned}
& \alpha_{k}(1)= \sum_{j \neq N} \frac{2 \sin (\pi j(2 k-1) / N) \sin (\pi j / N)}{(2-2 \cos (2 \pi j / N))^{3 / 2}} \\
&=\frac{1}{4} \sum_{j \neq N}\left[-\frac{1}{\sin (\pi j / N)}\right. \\
&\left.+\frac{2}{\sin (\pi j / N)} \sum_{m=0}^{k-1} \cos \frac{2 \pi j}{N} m\right] .
\end{aligned}
$$

Then, we get that

$$
\begin{aligned}
& \left(\alpha_{k}(1)-\alpha_{k-1}(1)\right)-\left(\alpha_{k+1}(1)-\alpha_{k}(1)\right) \\
& \quad=\frac{1}{2} \sum_{j \neq N} \frac{\cos (2 \pi j(k-1) / N)-\cos (2 \pi j k / N)}{\sin (\pi j / N)} \\
& =\sum_{j \neq N} \sin \frac{\pi j(2 k-1)}{N} \\
& =\cot \frac{\pi(2 k-1)}{2 N} .
\end{aligned}
$$

Notice that $0<\pi(2 k-1) /(2 N)<\pi / 2$ for $k=2, \ldots, \ell-1$. Hence, $\cot \pi(2 k-1) / 2 N>0$ for $k=2, \ldots, \ell-1$. Then we have

$$
\alpha_{k}(1)-\alpha_{k-1}(1)>\alpha_{k+1}(1)-\alpha_{k}(1) \text {. }
$$

By (52), we get that $\alpha_{k-1}(1)+\alpha_{\mathrm{k}+1}(1)<2 \alpha_{k}(1)$ which implies that $\alpha_{k+1}(1)<\alpha_{k}(1)$ or $\alpha_{k-1}(1)<\alpha_{k}(1)$. If there is $\alpha_{k}(1) \leq 0$, we use two cases to proceed our proof.

Case 1. (If $\alpha_{k-1}(1)<\alpha_{k}(1)$ ). By (52), we have $\alpha_{k-2}(1)-$ $\alpha_{k-1}(1)<\alpha_{k-1}(1)-\alpha_{k}(1)<0$ and hence $\alpha_{k-2}(1)<\alpha_{k-1}(1)<$ $\alpha_{k}(1) \leq 0$. By inductions, we have $\alpha_{1}(1)<\cdots<\alpha_{k}(1)<0$ which contradicts with $\alpha_{1}(1)>0$.

Case 2. (If $\alpha_{k+1}$ (1) $<\alpha_{k}(1)$ ). By (52), we have $\alpha_{k+2}(1)-$ $\alpha_{k+1}(1)<\alpha_{k+1}(1)-\alpha_{k}(1)<0$. Then, $\alpha_{k+2}(1)<\alpha_{k+1}(1)<$ $\alpha_{k}(1) \leq 0$. By inductions, we get that for even $N, \alpha_{N / 2}(1)<$ $\alpha_{(N / 2)-1}(1)<\cdots<\alpha_{k}(1)<0$ which contradicts with $\alpha_{N / 2}(1)>0$; for odd $N, \alpha_{(N+1) / 2}(1)<\alpha_{(N-1) / 2}(1)<\cdots<$ $\alpha_{k}(1) \leq 0$ which contradicts with $\alpha_{(N+1) / 2}(1)=0$.
From Cases 1 and 2, we see that there is always a contradiction if there exists $\alpha_{k}(1) \leq 0$. Hence, $\alpha_{k}(1)>0, k=1$, $\ldots, \ell$.

Let

$$
\begin{aligned}
& \tilde{\ell}= \begin{cases}\frac{N}{2}, & \text { if } N \text { is even, } \\
\frac{(N-1)}{2}, & \text { if } N \text { is odd, }\end{cases} \\
& \widehat{a}^{*}=\min _{a \in(0,1), \alpha_{k}(a)>0, k=1,2, \ldots, \tilde{\ell}} a .
\end{aligned}
$$

By the continuity, Lemma 14 and Proposition 15, we see that $\widehat{a}^{*} \in(0,1)$.

For $k=1,2, \ldots, \ell$, let

$$
\beta_{k}(a)=\sum_{j \neq N} \frac{\cos (2 \pi j(k-1) / N)-\cos (2 \pi j k / N)}{\left|1-a \rho_{j}\right|^{5}} .
$$

Similar to Lemma 14, we have

Lemma 16. If $N$ is odd, then $\beta_{(N+1) / 2}(1)=0$; If $N$ is even, then $\beta_{N / 2}(1)>0$.

Proposition 17. For $k=1,2, \ldots, \ell-1$, then $\beta_{k}(1)>0$.

Proof. Clearly, $\beta_{1}(1)>0$. We need to prove $\beta_{k}(1)>0, k \geq 2$. Note that

$$
\begin{aligned}
& \frac{2}{\sin ^{3}(\pi j / N)} \sum_{m=0}^{k-1} \cos \frac{2 \pi j}{N} m \\
& =\frac{1}{\sin ^{3}(\pi j / N)} \cdot \frac{2 \sin (\pi j k / N) \cos (\pi j(k-1) / N)}{\sin (\pi j / N)} \\
& =\frac{\sin (\pi j(2 k-1) / N)}{\sin ^{4}(\pi j / N)}+\frac{1}{\sin ^{3}(\pi j / N)} .
\end{aligned}
$$

By the definition of $\beta_{k}(1)$, we have

$$
\begin{aligned}
\beta_{k}(1)=\frac{1}{16} \sum_{j \neq N}[- & \frac{1}{\sin ^{3}(\pi j / N)} \\
& \left.+\frac{2}{\sin ^{3}(\pi j / N)} \sum_{m=0}^{k-1} \cos \frac{2 \pi j}{N} m\right] .
\end{aligned}
$$

Then, we get that

$$
\begin{aligned}
& \left(\beta_{k}(1)-\beta_{k-1}(1)\right)-\left(\beta_{k+1}(1)-\beta_{k}(1)\right) \\
& \quad=\frac{1}{8} \sum_{j \neq N} \frac{\cos (2 \pi j(k-1) / N)-\cos (2 \pi j k / N)}{\sin ^{3}(\pi j / N)} \\
& \quad=\frac{1}{8} \alpha_{k}(1)>0 .
\end{aligned}
$$

By similar proofs as in Proposition 15 , we can get $\beta_{k}>0$. The proof is completed. 
Let

$$
\tilde{a}^{*}=\min _{a \in(0,1), \beta_{k}(a)>0, k=1,2, \ldots, \tilde{e}} a .
$$

By the continuity, Lemma 16 and Proposition 17, we see that $\tilde{a}^{*} \in(0,1)$.

Lemma 18. For $k=2,3, \ldots, \ell$ and $a^{*}=\max \left\{\hat{a}^{*}, \widetilde{a}^{*}\right\}$, $\lambda_{k}^{(1)} \lambda_{k}^{(4)}-\lambda_{k}^{(2)} \lambda_{k}^{(3)}>0$.

Proof. Let $A_{k}(a)=\lambda_{k}^{(1)} \lambda_{k}^{(4)}-\lambda_{k}^{(2)} \lambda_{k}^{(3)}$. From (34) and (35), we can get

$$
\begin{aligned}
A_{k}(a)= & \frac{1}{a^{2}}\left(\sum_{j \neq N} \frac{\cos (2 \pi j(k-1) / N)-\cos (2 \pi j k / N)}{\left|1-\rho_{j}\right|^{3}}\right)^{2} \\
& -\left(\sum \frac{\cos (2 \pi j(k-1) / N)-a \cos (2 \pi j k / N)}{\left|1-a \rho_{j}\right|^{3}}\right) \\
& \cdot\left(\sum \frac{a \cos (2 \pi j(k-1) / N)-\cos (2 \pi j k / N)}{\left|a-\rho_{j}\right|^{3}}\right) .
\end{aligned}
$$

By the definitions of $\ell$ and $\tilde{\ell}$, we see that $\tilde{\ell}=\ell$ if $N$ is even and $\tilde{\ell}=\ell-1$ if $N$ is odd. For odd $N$, we get

$$
\begin{aligned}
& A_{(N+1) / 2}(a) \\
& =\frac{1}{a^{2}}\left(\sum _ { j \neq N } \left(\cos \frac{2 \pi j(((N+1) / 2)-1)}{N}\right.\right. \\
& \left.\left.-\cos \frac{2 \pi j((N+1) / 2)}{N}\right) \times\left|1-\rho_{j}\right|^{-3}\right)^{2} \\
& -\left(\sum \left(\cos \frac{2 \pi j(((N+1) / 2)-1)}{N}\right.\right. \\
& \left.\left.-a \cos \frac{2 \pi j((N+1) / 2)}{N}\right) \times\left|1-a \rho_{j}\right|^{-3}\right) \\
& \cdot\left(\sum \left(a \cos \frac{2 \pi j(((N+1) / 2)-1)}{N}\right.\right. \\
& \left.\left.-\cos \frac{2 \pi j((N+1) / 2)}{N}\right) \times\left|a-\rho_{j}\right|^{-3}\right) \\
& =(1-a)^{2}\left[\sum(-1)^{j} \frac{\cos (\pi j / N)}{\left|1-a \rho_{j}\right|^{3}}\right]^{2} .
\end{aligned}
$$

Let $f(x)=\cos x /\left(1+a^{2}-2 a \cos 2 x\right)^{3 / 2}$ for $0<x<\pi / 2$. Then

$$
f^{\prime}(x)=\frac{-\sin x\left(1+a^{2}-2 a \cos 2 x\right)-6 a \cos x \sin 2 x}{\left(1+a^{2}-2 a \cos 2 x\right)^{5 / 2}}<0 .
$$

Hence, $\cos (\pi j / N)(a-1) /\left|a-\rho_{j}\right|^{3}$ is decreasing in $j$ for $0<$ $\pi j / N<\pi / 2$. Note that the signs of the terms alternate, then the summation is negative for $0<\pi j / N<\pi / 2$. By symmetry, the summation for the rest terms is also negative. Hence, we have

$$
A_{(N+1) / 2}(a)>0 .
$$

For any $k, k=2,3, \ldots, \tilde{\ell}$, let

$$
\begin{aligned}
G(a)= & \sum_{j \neq N} \frac{\cos (2 \pi j(k-1) / N)-\cos (2 \pi j k / N)}{\left|1-\rho_{j}\right|^{3}} \\
& -a^{3 / 2} \sum_{j \neq N} \frac{\cos (2 \pi j(k-1) / N)-\cos (2 \pi j k / N)}{\left|1-a \rho_{j}\right|^{3}} .
\end{aligned}
$$

With direct computation, we have

$$
\begin{aligned}
& G^{\prime}(a)= \frac{3}{2} a^{1 / 2}(a+1)(a-1) \\
& \times \sum_{j \neq N} \frac{\cos (2 \pi j(k-1) / N)-\cos (2 \pi j k / N)}{\left|1-a \rho_{j}\right|^{5}}<0, \\
& \quad \text { for } a \in\left(a^{*}, 1\right),
\end{aligned}
$$

where Proposition 17 is used. It follows from (64) that $G(a)$ is decreasing for $a \in\left(a^{*}, 1\right)$. Note that $G(1)=0$. So, $G(a)>$ $G(1)=0$ for $a \in(0,1)$. It is that

$$
\begin{aligned}
& \sum_{j \neq N} \frac{\cos (2 \pi j(k-1) / N)-\cos (2 \pi j k / N)}{\left|1-\rho_{j}\right|^{3}} \\
& >a^{3 / 2} \sum_{j \neq N} \frac{\cos (2 \pi j(k-1) / N)-\cos (2 \pi j k / N)}{\left|1-a \rho_{j}\right|^{3}}
\end{aligned}
$$

where $a \in\left(a^{*}, 1\right)$ and $k=2,3, \ldots, \tilde{\ell}$. From $(65)$, we can get

$$
\begin{gathered}
\frac{1}{a^{2}}\left(\sum_{j \neq N} \frac{\cos (2 \pi j(k-1) / N)-\cos (2 \pi j k / N)}{\left|1-\rho_{j}\right|^{3}}\right)^{2} \\
>a\left(\sum_{j \neq N} \frac{\cos (2 \pi j(k-1) / N)-\cos (2 \pi j k / N)}{\left|1-a \rho_{j}\right|^{3}}\right)^{2},
\end{gathered}
$$

where $a \in\left(a^{*}, 1\right)$ and $k=2,3, \ldots, \tilde{\ell}$. 
By (59), we can get

$$
\begin{aligned}
A_{k}(a)= & \frac{1}{a^{2}}\left(\sum_{j \neq N} \frac{\cos (2 \pi j(k-1) / N)-\cos (2 \pi j k / N)}{\left|1-\rho_{j}\right|^{3}}\right)^{2} \\
& -a\left(\sum \frac{\cos (2 \pi j(k-1) / N)}{\left|1-a \rho_{j}\right|^{3}}\right)^{2} \\
& +\left(1+a^{2}\right)\left(\sum \frac{\cos (2 \pi j(k-1) / N)}{\left|1-a \rho_{j}\right|^{3}}\right) \\
& \times\left(\sum \frac{\cos (2 \pi j k / N)}{\left|1-a \rho_{j}\right|^{3}}\right)^{2} \\
& -a\left(\sum \frac{\cos (2 \pi j k / N)}{\left|1-a \rho_{j}\right|^{3}}\right)^{2}
\end{aligned}
$$

From Proposition 12, we have

$$
\begin{aligned}
& A_{k}(a)>\frac{1}{a^{2}}\left(\sum_{j \neq N} \frac{\cos (2 \pi j(k-1) / N)-\cos (2 \pi j k / N)}{\left|1-\rho_{j}\right|^{3}}\right)^{2} \\
& -a\left\{\left(\sum \frac{\cos (2 \pi j(k-1) / N)}{\left|1-a \rho_{j}\right|^{3}}\right)^{2}\right. \\
& -2\left(\sum \frac{\cos (2 \pi j(k-1) / N)}{\left|1-a \rho_{j}\right|^{3}}\right) \\
& \times\left(\sum \frac{\cos (2 \pi j k / N)}{\left|1-a \rho_{j}\right|^{3}}\right) \\
& \left.+\left(\sum \frac{\cos (2 \pi j k / N)}{\left|1-\mathrm{a} \rho_{j}\right|^{3}}\right)^{2}\right\} \\
& =\frac{1}{a^{2}}\left(\sum_{j \neq N} \frac{\cos (2 \pi j(k-1) / N)-\cos (2 \pi j k / N)}{\left|1-\rho_{j}\right|^{3}}\right)^{2} \\
& -a\left(\sum_{j \neq N} \frac{\cos (2 \pi j(k-1) / N)-\cos (2 \pi j k / N)}{\left|1-a \rho_{j}\right|^{3}}\right)^{2}
\end{aligned}
$$$$
>0
$$

where (66) is used and $k=2,3, \ldots, \tilde{\ell}$.

By (62) and (68), the proof is completed.

The following example illustrate that the $a^{*}$ in Lemma 18 can be obtained for some special cases.
Example 19. If $N=3$, then $a^{*}=0$.

Proof. For $N=3$, we have

$$
\begin{aligned}
& \alpha_{1}(a)=\frac{2}{\left((1+(a / 2))^{2}+(3 / 4)\right)^{3 / 2}}>0, \\
& \alpha_{2}(a)=\frac{1}{\left((1+(a / 2))^{2}+(3 / 4)\right)^{3 / 2}}>0 .
\end{aligned}
$$

By the definition of $\widehat{a}^{*}$, we have $\widehat{a}^{*}=0$.

Similarly, we get that

$$
\begin{aligned}
& \beta_{1}(a)=\frac{2}{\left((1+(a / 2))^{2}+(3 / 4)\right)^{5 / 2}}>0, \\
& \beta_{2}(a)=\frac{1}{\left((1+(a / 2))^{2}+(3 / 4)\right)^{5 / 2}}>0 .
\end{aligned}
$$

By the definition of $\tilde{a}^{*}$, we have $\tilde{a}^{*}=0$. Hence, $a^{*}=0$ for $N=3$.

Lemmas 13 and 18 show that the hypotheses of Lemma 9 are satisfied. Thus the proof of the Theorem 5 was completed.

\section{The Proof of Theorem 6}

Since $m_{1}=m_{2}=\cdots=m_{N}=m$ and $\widetilde{m}_{1}=\widetilde{m}_{2}=\cdots=\widetilde{m}_{N}=$ $\widetilde{m}$, we get that $z_{0}=0$. Hence, by (3), we have

$$
q_{k}=\rho_{k} e^{i \omega t}, \quad \tilde{q}_{k}=a \rho_{k} e^{i \omega t} .
$$

Substituting $q_{k}$ and $\tilde{q}_{k}$ into (1), then by (11) we get that

$$
\begin{aligned}
\omega^{2} & =\frac{m}{4} \sum_{j \neq N} \csc \frac{\pi j}{N}+\widetilde{m} \sum \frac{1-a \cos (2 \pi j / N)}{\left|1-a \rho_{j}\right|^{3}}, \\
a \omega^{2} & =m \sum \frac{a-\cos (2 \pi j / N)}{\left|1-a \rho_{j}\right|^{3}}+\frac{\widetilde{m}}{4 a^{2}} \sum_{j \neq N} \csc \frac{\pi j}{N} .
\end{aligned}
$$

From (72), we have

$$
\begin{gathered}
\omega^{2}=\frac{m}{4} \sum_{j \neq N} \csc \frac{\pi j}{N}+\widetilde{m} \sum \frac{1-a \cos (2 \pi j / N)}{\left|1-a \rho_{j}\right|^{3}}, \\
\omega^{2}=\frac{1}{a}\left[m \sum \frac{a-\cos (2 \pi j / N)}{\left|1-a \rho_{j}\right|^{3}}+\frac{\widetilde{m}}{4 a^{2}} \sum_{j \neq N} \csc \frac{\pi j}{N}\right] .
\end{gathered}
$$

From (73), the constant $\omega^{2}$ exists only if the following equation holds:

$$
\begin{gathered}
a\left\{\frac{m}{4} \sum_{j \neq N} \csc \frac{\pi j}{N}+\widetilde{m} \sum \frac{1-a \cos (2 \pi j / N)}{\left|1-a \rho_{j}\right|^{3}}\right\} \\
=m \sum \frac{a-\cos (2 \pi j / N)}{\left|1-a \rho_{j}\right|^{3}}+\frac{\widetilde{m}}{4 a^{2}} \sum_{j \neq N} \csc \frac{\pi j}{N} .
\end{gathered}
$$


Note that $\mu=\widetilde{m} / m$. Then we get from (74) that

$$
\frac{a^{3}-\mu}{4 a^{2}} \sum_{j \neq N} \csc \frac{\pi j}{N}-(1-\mu) a f(a)+\left(1-a^{2} \mu\right) g(a)=0,
$$

where $f(a)=\sum\left(1 /\left|1-a \rho_{j}\right|^{3}\right)$ and $g(a)=\sum(\cos (2 \pi j / N) / \mid 1-$ $\left.\left.a \rho_{j}\right|^{3}\right)$.

Lemma 20. For $\mu>0$ and $N \geq 3$, the constant $\omega^{2}$ can be given by

$$
\omega^{2}=\frac{m}{4} \sum_{j \neq N} \csc \frac{\pi j}{N}+\widetilde{m} \sum \frac{1-a_{0} \cos (2 \pi j / N)}{\left|1-a_{0} \rho_{j}\right|^{3}},
$$

where $a_{0}$ is the unique solution of (75).

Proof. By the relationship between (74) and (75), it suffices to prove that (75) has solution $a=a_{0}$ for $\mu>0$ and $N \geq 3$. Let

$$
\begin{aligned}
F(a)= & \left(a-\frac{\mu}{a^{2}}\right) \frac{1}{4} \sum_{j \neq N} \csc \frac{\pi j}{N} \\
& -(1-\mu) \text { af }(a)+\left(1-a^{2} \mu\right) g(a) .
\end{aligned}
$$

From (75) and (77), the roots of (75) are zeros of (77). From the definitions of $\psi_{\beta, k}(a)$ defined in Proposition 12, we have $\psi_{1, N}(a)=\sum\left(1 /\left|1-a \rho_{j}\right|\right)$. Through direct calculations, we find that

$$
\begin{aligned}
\psi_{1, N}(a) & =\left(1+a^{2}\right) f(a)-2 a g(a), \\
\frac{d \psi_{1, N}(a)}{d a} & =-a f(a)+g(a) .
\end{aligned}
$$

From (78), (77) can be written as

$$
\begin{aligned}
F(a)= & \left(a-\frac{\mu}{a^{2}}\right) \frac{1}{4} \sum_{j \neq N} \csc \frac{\pi j}{N} \\
& +\left(1+a^{2} \mu\right) \frac{d \psi_{1, N}(a)}{d a}+\mu a \psi_{1, N}(a) .
\end{aligned}
$$

Notice that $\psi_{1, N}(a)=\sum_{j \neq N}\left(1 /\left|1-a \rho_{j}\right|\right)+\left(1 /\left|1-a \rho_{N}\right|\right)=$ $\sum_{j \neq N}\left(1 /\left|1-a \rho_{j}\right|\right)+(1 /|1-\mathrm{a}|)$. Hence, we have

$$
\lim _{a \rightarrow 1} \psi_{1, N}(a)=+\infty \text {. }
$$

From (79), (80), and Proposition 12, we can get that

$$
\lim _{a \rightarrow 0} F(a)=-\infty, \quad \lim _{a \rightarrow 1} F(a)=+\infty .
$$

On the other hand, by (79), we get that

$$
\begin{aligned}
F^{\prime}(a)= & \left(1+\frac{2 \mu}{a^{3}}\right) \frac{1}{4} \sum_{j \neq N} \csc \frac{\pi j}{N} \\
& +\mu \psi_{1, N}(a)+3 a \mu \frac{d \psi_{1, N}(a)}{d a} \\
& +\left(1+a^{2} \mu\right) \frac{d^{2} \psi_{1, N}(a)}{d a^{2}} .
\end{aligned}
$$

From (82) and Proposition 12, we know that $F^{\prime}(a)>0$ for $a \epsilon$ $(0,1)$. This, together with $(81)$, implies that there is a unique solution $a=a_{0}$ for $F(a)=0$. The proof is completed.

\section{Acknowledgments}

The authors thank Professor Zhang S.Q. for his guidances, Editor Zheng B.D. for his hard work, and the anonymous referees for their comments, suggestions and, pointing out some additional references. Supported partially by Fundamental Research Funds for the Central Universities, CQDXWL2012-006.

\section{References}

[1] L. M. Perko and E. L. Walter, "Regular polygon solutions of the $N$-body problem," Proceedings of the American Mathematical Society, vol. 94, no. 2, pp. 301-309, 1985.

[2] R. Moeckel and C. Simó, "Bifurcation of spatial central configurations from planar ones," SIAM Journal on Mathematical Analysis, vol. 26, no. 4, pp. 978-998, 1995.

[3] S. Zhang and Q. Zhou, "Periodic solutions for planar N-body problems," Proceedings of the American Mathematical Society, vol. 131, no. 7, pp. 2161-2170, 2003.

[4] M. Marcus and H. Minc, A Survey of Matrix Theory and Matrix Inequalities, Allyn and Bacon, Boston, Mass, USA, 1964. 


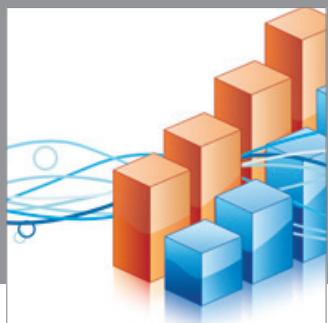

Advances in

Operations Research

mansans

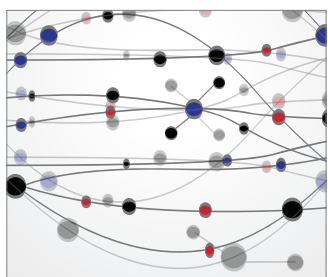

The Scientific World Journal
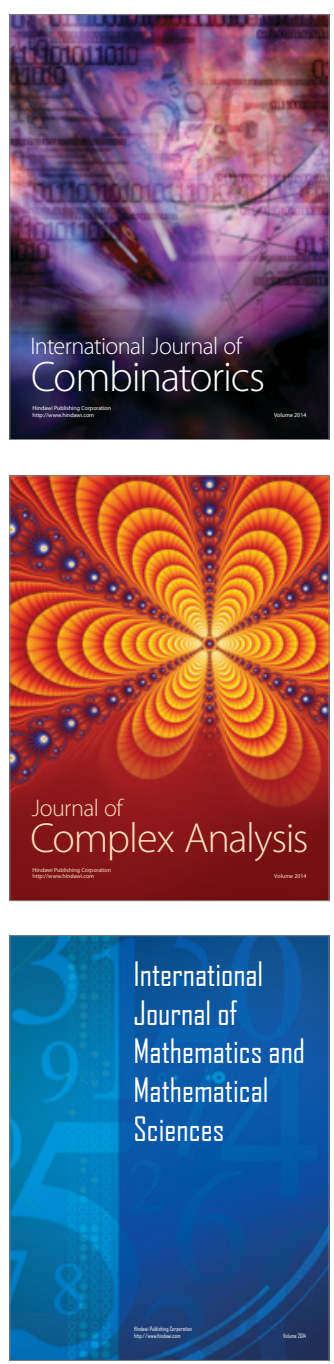
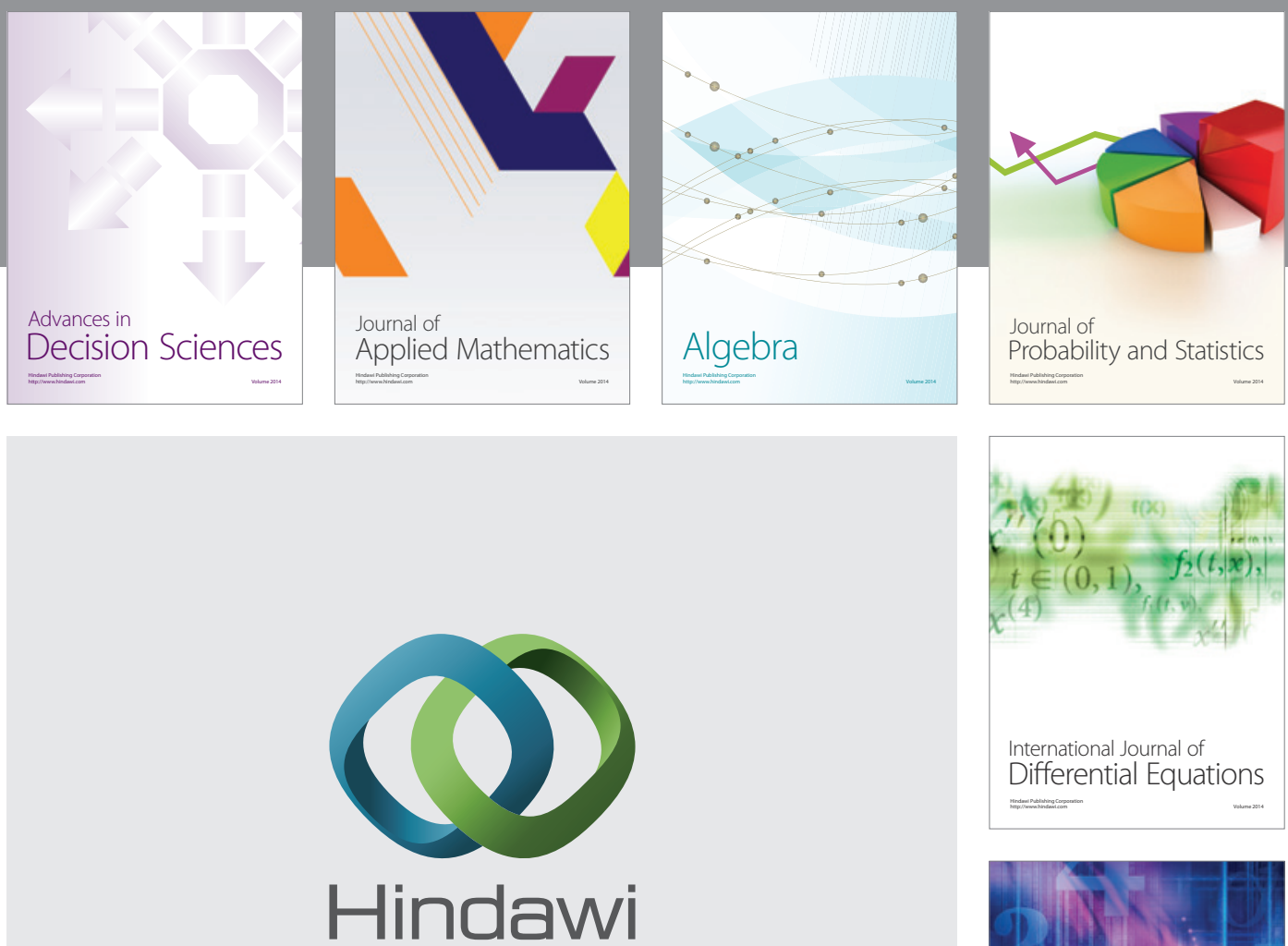

Submit your manuscripts at http://www.hindawi.com
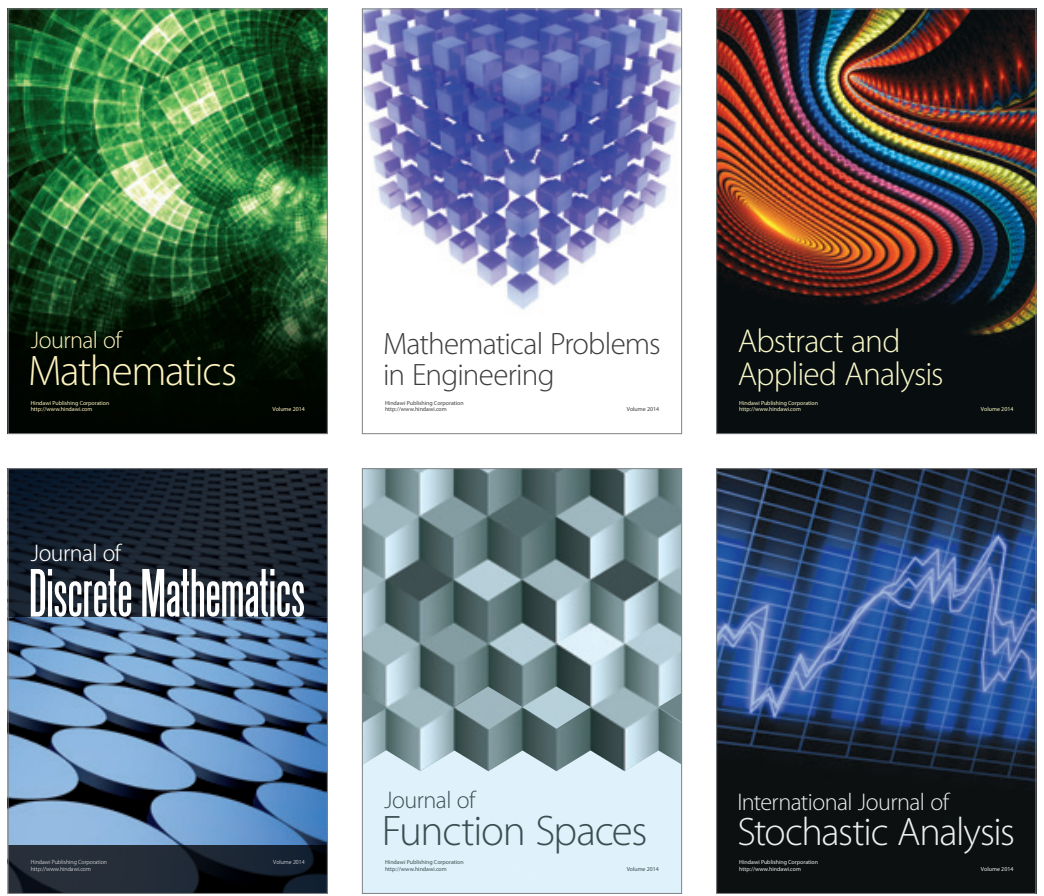

Journal of

Function Spaces

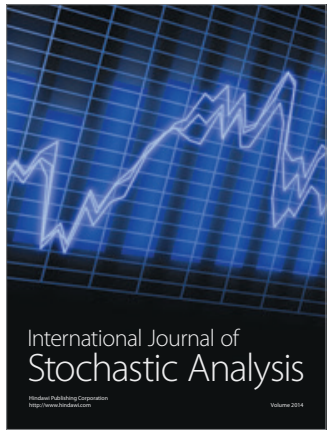

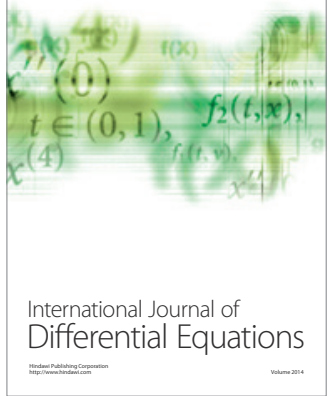
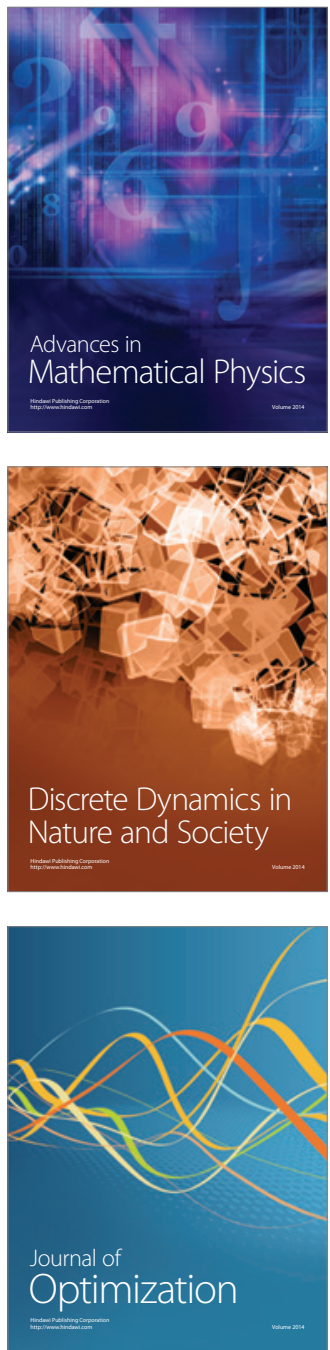